\title{
Frontier and Semifrontier Sets in Intuitionistic Topological Spaces
}

\author{
S. Girija ${ }^{1, *}$, S. Selvanayaki ${ }^{2}$, Gnanambal Ilango $^{3}$ \\ ${ }^{1}$ Hindusthan College of Engineering and Technology, Coimbatore,India. \\ ${ }^{2}$ Kumaraguru College of Technology,Coimbatore, India. \\ ${ }^{3}$ Government Arts College, Coimbatore,India.
}

\section{Abstract}

The notions of frontier and semifrontier in intuitionistic topology have been studied and several of their properties are proved. Many counter examples have been pointed out for the relevant classifications.

Received on 07 May 2018; accepted on 12 July 2018; published on 12 September 2018

Keywords: Intuitionistic frontier, Intuitionistic semifrontier, Intuitionistic semi continuous.

Copyright (c) 2018 S. Girija et al., licensed to EAI. This is an open access article distributed under the terms of the Creative Commons Attribution license (http://creativecommons.org/licenses/by/3.0/), which permits unlimited use, distribution and reproduction in any medium so long as the original work is properly cited.

3rd International Conference on Green, Intelligent Computing and Communication Systems - ICGICCS 2018, 18.5 - 19.5.2018, Hindusthan College of Engineering and Technology, India

doi:10.4108/eai.12-9-2018.155558

\section{Introduction}

Atanassov [1], in 1986, established the fundamentals of intuitionistic fuzzy set as a generalization of fuzzy sets of Zadeh [12] on the degree of membership and non membership. The fundamentals of intuitionistic topological spaces was instigated by Coker[4], in the year 2000. Intuitionistic sets (IS's) have been applied in areas of science and technology. Salama [8] has used intuitionistic topology (IT) for studying land cover changes. Considering the inherent nature of Geographic Information Science (GIS) phenomina, it seems more suitable to study the problem of land cover changes using intuitionistic fuzzy topology. For recasting the GIS problem in terms of intuitionistic topology, the study of intuitionistic frontier is necessary.

This paper provides the notion of intuitionistic frontier and its properties in intuitionistic topological spaces ITS $(X)$. By intuitionistic semiopen sets [5], the notion of intuitionistic semifrontier is defined and we characterize intuitionistic semi continuous functions with reference to intuitionistic semi frontier. Counter examples given herein are constructed upon the intuitionistic topological space defined by Coker[4].

*S.Girija. Email: girija6271@gmail.com

\section{Intuitionistic Frontier}

Definition : 2.1.[2] Consider a nonempty set as $X_{1}$. An IS $\mathrm{A}$, having the form $A=<X_{1}, A_{1}, A_{2}>$, where $A_{1}$ and $A_{2}$ are subsets of $X_{1}$ satisfying $A_{1} \cap A_{2}=\phi$. The set of members of $\mathrm{A}$ is $A_{1}$, and the set of non members is $A_{2}$. The set of all ITS in $X_{1}$ is denoted as $\operatorname{ITS}\left(X_{1}\right)$.

Definition : 2.2.[4] The nonempty set $X_{1}$ and $A, B$ are IS's in the form $\mathrm{A}=<X_{1}, A_{1}, A_{2}>, \mathrm{B}=<X_{1}, B_{1}, B_{2}>$ respectively. Then

(a) $\mathrm{A} \subseteq \mathrm{B}$ iff $A_{1} \subseteq B_{1}$ and $A_{2} \supseteq B_{2}$.

(b) $\mathrm{A}=\mathrm{B}$ iff $\mathrm{A} \subseteq \mathrm{B}$ and $\mathrm{B} \subseteq \mathrm{A}$.

(c) $\bar{A}=<X_{1}, A_{2}, A_{1}>$.

(d) $A-B=A \cap \bar{B}$.

(e) $\phi=<X_{1}, \phi, X_{1}>, \underset{\sim}{X}=<X_{1}, X_{1}, \phi>$.

(f) $\mathrm{A} \cup \mathrm{B}=<X_{1}, A_{1} \cup B_{1}, A_{2} \cap B_{2}>$.

(g) $\mathrm{A} \cap \mathrm{B}=<X_{1}, A_{1} \cap B_{1}, A_{2} \cup B_{2}>$.

Definition : 2.3.[4] An IT on a nonempty set $X_{1}$ is a family $\tau$ of IS's in $X_{1}$ satisfying the following axioms:

(a) $\phi, X \in \tau$

(b) $\tilde{G}_{1} \cap G_{2} \in \tau$ for any $G_{1}, G_{2} \in \tau$

(c) $\cup G_{i} \in \tau$ for any arbitrary family $\left\{G_{i}: i \in J\right\} \subseteq \tau$. In this case, the pair $(X, \tau)$ is called an intuitionistic topological space (ITS for short) and any intuitionistic set in $\tau$ is known as an intuitionistic open set (IOS for short) in $X$.

Definition : 2.4. [4] Let $(X, \tau)$ be an intuitionistic topological space $(\operatorname{ITS}(X))$ and $A=<X, A_{1}, A_{2}>$ be an IS in $X$. Then the interior and closure of $A$ are defined 
by

$\operatorname{Icl}(A)=\cap\{K: K$ is an ICS in $X$ and $A \subseteq K\}$

$\operatorname{Iint}(A)=\cup\{G: G$ is an IOS in $X$ and $G \subseteq A\}$.

It can be shown that $\operatorname{Icl}(A)$ is an $\operatorname{ICS}$ and $\operatorname{Iint}(A)$ is an IOS in X and $\mathrm{A}$ is an ICS in $\mathrm{X}$ iff $\operatorname{Icl}(A)=A$ and is an IOS in X iff $\operatorname{Iint}(A)=A$.

Definition : 2.5.[2] (a) Let $X$ be a nonempty set and $\mathrm{p} \in \mathrm{X}$, a fixed element in $\mathrm{X}$. Then the IS $p$ defined by $p=<X,\{p\},\{p\}^{c}>$ is called an intuitionistic point (IP for short) in X.

(b) Let $p$ be an IP in X and $\mathrm{A}=<X, A_{1}, A_{2}>$ an IS in X. Then $p$ is said to be contained in $\mathrm{A}$ ( $p \in A$ for short) if and only if $\mathrm{p} \in A_{1}$.

Definition : 2.6.[4] (a) Let $(X, \tau)$ and $(Y, \Phi)$ be two ITS's and let $f: X \rightarrow Y$ be a function. Then $\mathrm{f}$ is said to be continuous iff the preimage of each IS in $\Phi$ is an IS in $\tau$. (b) Let $(X, \tau)$ and $(Y, \Phi)$ be two ITS's and let $f: X \rightarrow Y$ be a function. Then $f$ is said to be open iff the image of each IS in $\tau$ is an IS in $\Phi$.

Definition : 2.7.[5] Let $(X, \tau)$ be an $\operatorname{ITS}(X)$. An intuitionistic set $\mathrm{A}$ of $\mathrm{X}$ is said to be intuitionistic semiopen if $A \subseteq \operatorname{Icl}(\operatorname{Iint}(A))$. The collection of all intuitionistic semiopen sets are denoted by $\operatorname{ISOS}(X)$. The complement of every intuitionistic semiopen set is intuitionistic semiclosed set and the collection of all intuitionistic sets are denoted by ISCS $(X)$.

Definition : 2.8.[5] Let $(X, \tau)$ be an intuitionistic topological space and $A=<X, A_{1}, A_{2}>$ be an IS in X. Then the intuitionistic semiinterior and intuitionistic semiclosure of $A$ are defined by

Iscl $(A)=\cap\{K: K$ is an ISCS in $X$ and $A \subseteq K\}$

Isint $(A)=\cup\{G: G$ is an ISOS in $X$ and $G \subseteq A\}$.

It can be shown that $\operatorname{Iscl}(A)$ is an ISCS and $\operatorname{Isint}(A)$ is an ISOS in $\mathrm{X}$ and $\mathrm{A}$ is an ISCS in $\mathrm{X}$ iff $\operatorname{Iscl}(A)=A$ and $\mathrm{A}$ is an ISOS in X iff Isint $(A)=A$.

Definition : 2.9.[4] A, B, C and $A_{i}$ be intuitionistic sets in $X(i \in J)$. Subsequently

(a) $A \subseteq B$ and $B \subseteq C \Rightarrow A \subseteq C$

(b) $A_{i} \subseteq B$ for each $i \in J \Rightarrow \cup A_{i} \subseteq B$

(c) $B \subseteq A_{i}$ for each $i \in J \Rightarrow B \subseteq \cap A_{i}$

(d) $\left(\cup A_{i}\right)^{c}=\cap A_{i}^{c}$

(e) $\left(\cap A_{i}\right)^{c}=\cup A_{i}^{c}$

( $f A \subseteq B \Leftrightarrow B^{c} \subseteq A^{c}$

$(g)\left(A^{c}\right)^{c}=A$

$\left(h(\phi)^{c}=X\right.$ and

(i) $(\underset{\sim}{X})^{c}=\phi$

Definition : 2.10.[11] Let $(X, \tau)$ and $(Y, \Phi)$ be two intuitionistic topological spaces and let $f: X \rightarrow Y$ be a function. Then $f$ is said to be intuitionistic semi continuous, if the inverse image of every intuitionistic open set of $(Y, \Phi)$ is intuitionistic semi open in $(X, \tau)$.

Proposition : 2.11.[4] Let $X$ be a nonempty set and let $\mathrm{A}, \mathrm{B}$ are intuitionistic sets in the form $\mathrm{A}=\left\langle X, A_{1}, A_{2}\right\rangle$,
$\mathrm{B}=<X, B_{1}, B_{2}>$ respectively. Then
(a) $\operatorname{Icl}(A \cup B)=\operatorname{Icl}(A) \cup \operatorname{Icl}(B)$
(b) $\operatorname{Iint}(A \cap B)=\operatorname{Iint}(A) \cap \operatorname{Iint}(B)$
(c) $\operatorname{Icl}(A \cap B) \subset \operatorname{Icl}(A) \cap \operatorname{Icl}(B)$
(d) $\operatorname{Icl}(A \cup B) \supset \operatorname{Icl}(A) \cup \operatorname{Icl}(B)$.

\section{Intuitionistic Frontier}

Definition: 3.1. Consider $\left(X_{1}, \mu\right)$ be an $\operatorname{ITS}\left(X_{1}\right)$ and $\mathrm{K}$ $\in \operatorname{IS}\left(X_{1}\right)$. Then $q \in \operatorname{IFr} P\left(X_{1}\right)$ is called an intuitionistic frontier point $\tilde{(}(\operatorname{IFr} P)$ of $\mathrm{K}$ if $q \in \operatorname{Icl}(\mathrm{K}) \cap \operatorname{Icl}\left(K^{c}\right)$. The union of all IFrPs of $K$ is termed as an IFrP of $\mathrm{K}$ and is represented by $\operatorname{IFr}(K)$. It is clear that $\operatorname{IFr}(K)=\operatorname{Icl}(K) \cap \operatorname{Icl}\left(K^{c}\right)$.

Proposition : 3.2. Each IS $M$ in $X_{1}, M \cup \operatorname{IFr}(M) \subset$ $\operatorname{Icl}(M)$.

Proof: Let $M$ be an IS in $X_{1}$ and $\operatorname{Icl}(M)=\left(X_{1}-\right.$ $\left.\operatorname{Icl}\left(X_{1}-M\right)\right)$ and $\operatorname{Icl}\left(M^{c}\right)=\operatorname{Icl}\left(X_{1}-M\right)$. Also $\operatorname{IFr}(\tilde{M})=$ $\operatorname{Icl}(\tilde{M}) \cap \operatorname{Icl}\left(M^{c}\right)=\left(X_{1}-\operatorname{Icl}\left(X_{1}-M\right)\right) \cap\left(\operatorname{Icl}\left(X_{1}-M\right)\right.$

$=\phi$. So $\operatorname{MUIFr}(\tilde{M})=M$. Since $\operatorname{M\subset Icrl}(M), \quad M$ $\cup \tilde{F r}(M) \subset \operatorname{Icl}(M)$.

Remark: 3.3. Equality concept cannot be replaced in Proposition 3.2.

Example:3.4. Consider $X_{1}=\left\{a_{1}, b_{1}, c_{1}\right\} \quad$ with intuitionistic topology $\mu=\left\{X_{1}, \phi,<X_{1},\left\{c_{1}\right\},\left\{b_{1}\right\}\right\rangle$, $\left.<X_{1},\left\{a_{1}, c_{1}\right\}, \phi>\right\}$. For $M=<X_{1},\left\{a_{1}, c_{1}\right\}, \phi>, \operatorname{Icl}(M)=$ $X_{1}$ and $\operatorname{IFr}(M)=<X_{1}, \phi,\left\{a_{1}, c_{1}\right\}>$.

But $\operatorname{M} \cup \operatorname{IFr}(M)=<X_{1},\left\{a_{1}, c_{1}\right\}, \phi>\neq \operatorname{Icl}(M)$.

Theorem:3.5. For an $\operatorname{ITS}\left(X_{1}, \mu\right)$, the following results hold.

(a) $\operatorname{IFr}(M)=\operatorname{IFr}\left(M^{c}\right)$.

(b) If $\mathrm{M}$ is an $\operatorname{ICS}$ then $\operatorname{IFr}(M) \subseteq M$.

(c) If $\mathrm{M}$ is an IOS then $\operatorname{IFr}(M) \subseteq M^{c}$.

$(d)(\operatorname{IFr}(M))^{c}=\operatorname{Iint}(M) \cup \operatorname{Iint}\left(M^{c}\right)$.

Proof: $(a) \operatorname{IFr}(M)=\operatorname{Icl}(M) \cap \operatorname{Icl}\left(M^{c}\right)=\operatorname{Icl}\left(M^{c}\right) \cap \operatorname{Icl}(M)$ $=\operatorname{Icl}\left(M^{c}\right) \cap \operatorname{Icl}\left(\left(M^{c}\right)\right)^{c}=\operatorname{IFr}\left(M^{c}\right)$.

(b) Considering as $\mathrm{M}$ be an ICS in $X_{1}, \operatorname{IFr}(M)=$ $\operatorname{Icl}(M) \cap \operatorname{Icl}\left(M^{c}\right) \subseteq \operatorname{Icl}(M)=M$. Hence $\operatorname{IFr}(M) \subseteq M$.

(c) $\mathrm{M}$ is an IOS implies $M^{c}$ is ICS. By $(b), \operatorname{IFr}\left(M^{c}\right) \subseteq M^{c}$, and by $(a) \operatorname{IFr}(M) \subseteq M^{c}$.

(d) $\quad(\operatorname{IFr}(M))^{c}=\left(\operatorname{Icl}(M) \cap \operatorname{Icl}\left(M^{c}\right)\right)^{c}=(\operatorname{Icl}(M))^{c} \cap$ $\left(\operatorname{Icl}\left(M^{c}\right)\right)^{c}=\operatorname{Iint}\left(M^{c}\right) \cup \operatorname{Iint}(M)$.

Remark:3.6. In general, the converse of $(b)$ and $(c)$ of Theorem 3.5 is not satisfied.

Example:3.7. Let $X_{1}=\left\{a_{1}, b_{1}, c_{1}\right\}$ with intuitionistic topology $\mu=\left\{X_{1}, \phi,<X_{1},\left\{a_{1}, b_{1}\right\},\left\{c_{1}\right\}>,<X_{1},\left\{a_{1}\right\}, \phi>\right.$,

$<X_{1},\left\{a_{1}, c_{1}\right\}, \phi>,<X_{1}, \phi,\left\{a_{1}, b_{1}\right\}>,<X_{1},\left\{a_{1}\right\},\left\{b_{1}\right\}>$,

$<X_{1}, \phi,\left\{c_{1}\right\}>,<X_{1},\left\{a_{1}, b_{1}\right\}, \phi>,<X_{1},\left\{a_{1}\right\},\left\{c_{1}\right\}>$,

$\left.<X_{1},\left\{a_{1}\right\},\left\{b_{1}, c_{1}\right\}>,\left\langle X_{1}, \phi, \phi\right\rangle,<X_{1},\left\{b_{1}\right\}, \phi\right\rangle$,

$<X_{1},\left\{b_{1}\right\},\left\{a_{1}\right\}>,<X_{1}, \phi,\left\{b_{1}, c_{1}\right\}>,<X_{1},\left\{c_{1}\right\},\left\{b_{1}\right\}>$,

$\left.<X_{1},\left\{a_{1}, c_{1}\right\},\left\{b_{1}\right\}>,<X_{1},\left\{b_{1}, c_{1}\right\},\left\{a_{1}\right\}>,<X_{1}, \phi,\left\{a_{1}\right\}\right\rangle$, 
$<X_{1},\left\{c_{1}\right\}, \phi><X_{1},\left\{c_{1}\right\},\left\{a_{1}, b_{1}\right\}>,<X_{1}, \phi,\left\{a_{1}, c_{1}\right\}>$, $\left.<X_{1}, \phi,\left\{b_{1}\right\}>\right\}$. (i) Let $\mathrm{M}=<X_{1},\left\{b_{1}\right\},\left\{a_{1}, c_{1}\right\}>$, then $\operatorname{IFr}(M)=<X_{1}, \phi,\left\{a_{1}, c_{1}\right\}>$ which implies $\operatorname{IFr}(M) \subseteq$ M but $\mathrm{M} \nsubseteq \operatorname{IFr}(M)$. (ii) Let $\mathrm{N}=<X_{1},\left\{a_{1}\right\},\left\{c_{1}\right\}>$, then $\operatorname{IFr}(N)$ $=\left\langle X_{1}, \phi,\left\{a_{1}, c_{1}\right\}\right\rangle$ which implies, $\operatorname{IFr}(N) \subseteq \mathrm{N}^{c}$ but $\mathrm{N}^{c} \nsubseteq \operatorname{IFr}(N)$.

Theorem:3.8. If $M$ and $N$ be IS's in an $\operatorname{ITS}\left(X_{1}\right)$, then $\operatorname{IFr}(M \cup N) \subseteq \operatorname{IFr}(M) \cup \operatorname{IFr}(N)$.

Proof:If $\operatorname{IFr}(M \cup N)=\operatorname{Icl}(M \cup N) \cap \operatorname{Icl}(M \cup N)^{c}$

$\subseteq(\operatorname{Icl}(M) \cup \operatorname{Icl}(N)) \cap\left(\operatorname{Icl}\left(M^{c}\right) \cap \operatorname{Icl}\left(N^{c}\right)\right)$

$\subseteq\left((\operatorname{Icl}(M) \cup \operatorname{Icl}(N)) \cap\left(\operatorname{Icl}\left(M^{c}\right)\right)\right) \cap((\operatorname{Icl}(M) \cup \operatorname{Icl}(N)) \cap$ $\left.\operatorname{Icl}\left(N^{c}\right)\right)$

$\subseteq\left(\left(\operatorname{Icl}(M) \cap \operatorname{Icl}\left(M^{c}\right)\right) \cup\left(\operatorname{Icl}(N) \cap \operatorname{Icl}\left(M^{c}\right)\right)\right) \cap((\operatorname{Icl}(M) \cap$

$\left.\left.\operatorname{Icl}\left(N^{c}\right)\right) \cup\left(\operatorname{Icl}(M) \cap \operatorname{Icl}\left(N^{c}\right)\right)\right)$

$\subseteq\left(\operatorname{IFr}(M) \cup\left(\operatorname{Icl}(N) \cap \operatorname{Icl}\left(M^{c}\right)\right)\right) \cap\left(\left(\operatorname{Icl}(M) \cap \operatorname{Icl}\left(N^{c}\right)\right) \cup\right.$ $\operatorname{IFr}(N))$

$\subseteq(\operatorname{IFr}(M) \cup \operatorname{IFr}(N)) \cap\left(\left(\operatorname{Icl}(N) \cap \operatorname{Icl}\left(M^{c}\right)\right) \cup(\operatorname{Icl}(M) \cap\right.$

$\left.\left.\operatorname{Icl}\left(N^{c}\right)\right)\right)$

$\subseteq \operatorname{IFr}(M) \cup \operatorname{IFr}(N)$

Converse of Theorem 3.8, does not hold.

Example:3.9. Let $X_{2}=\left\{a_{2}, b_{2}, c_{2}\right\}$ with intuitionistic topology $\mu=\left\{X_{2}, \phi,<X_{2},\left\{c_{2}\right\},\left\{b_{2}\right\}>,<X_{2},\left\{a_{2}, c_{2}\right\}, \phi>\right\}$

and let $A_{2}=<X_{2},\left\{a_{2}, c_{2}\right\}, \phi>, B_{2}=<X_{2},\left\{a_{2}, b_{2}\right\},\left\{c_{2}\right\}>$. Then $\left.\operatorname{IFr}\left(A_{2}\right)=<X_{2}, \phi,\left\{a_{2}, c_{2}\right\}\right\rangle, \operatorname{IFr}\left(B_{2}\right)=\left\langle X_{2}, X_{2}, \phi\right\rangle$, and $\operatorname{IFr}\left(A_{2} \cup B_{2}\right)=\left\langle X_{2}, \phi, X_{2}\right\rangle, \quad \operatorname{IFr}\left(A_{2}\right) \cup \operatorname{IFr}\left(B_{2}\right)=$ $\left.<X_{2}, X_{2}, \phi\right\rangle$ which implies $\operatorname{IFr}\left(A_{2} \cup B_{2}\right) \subseteq$ $\operatorname{IFr}\left(A_{2}\right) \cup \operatorname{IFr}\left(B_{2}\right)$ but $\operatorname{IFr}\left(A_{2}\right) \cup \operatorname{IFr}\left(B_{2}\right) \nsubseteq \operatorname{IFr}\left(A_{2} \cup B_{2}\right)$. Theorem:3.10. Let $A_{1}$ and $B_{1}$ be two IS's in an $\operatorname{ITS}\left(X_{1}\right)$, $\operatorname{IFr}\left(A_{1} \cap B_{1}\right) \subseteq\left(\operatorname{IFr}\left(A_{1}\right) \cap \operatorname{Icl}\left(B_{1}\right)\right) \cup\left(\operatorname{IFr}\left(B_{1}\right) \cap\right.$

$\left.\operatorname{Icl}\left(A_{1}\right)\right)$.

Proof:Consider $\operatorname{IFr}\left(A_{1} \cap B_{1}\right)=\operatorname{Icl}\left(A_{1} \cap B_{1}\right) \cap \operatorname{Icl}\left(A_{1} \cap\right.$ $\left.B_{1}\right)^{c}$

$\subseteq\left(\left(\operatorname{Icl}\left(A_{1}\right) \cap \operatorname{Icl}\left(B_{1}\right)\right) \cap\left(\operatorname{Icl}\left(A_{1}^{c}\right) \cup \operatorname{Icl}\left(B_{1}^{c}\right)\right)\right)$

$\subseteq\left(\left(\operatorname{Icl}\left(A_{1}\right) \cap \operatorname{Icl}\left(B_{1}\right)\right) \cap \operatorname{Icl}\left(A_{1}^{c}\right)\right) \cup\left(\left(\operatorname{Icl}\left(A_{1}\right) \cap \operatorname{Icl}\left(B_{1}\right)\right) \cap\right.$

$\left.\operatorname{Icl}\left(B_{1}^{c}\right)\right)$

$\subseteq\left(\operatorname{IFr}\left(A_{1}\right) \cap \operatorname{Icl}\left(B_{1}\right)\right) \cup\left(\operatorname{IFr}\left(B_{1}\right) \cap \operatorname{Icl}\left(A_{1}\right)\right)$.

The reverse process of Theorem 3.10. does not satisfied.

Example:3.11. Consider $X_{3}=\{11,22\} \quad$ with intuitionistic topology $\mu=\left\{X_{3}, \phi,<X_{3}, \phi,\{22\}>\right.$,

$\left.<X_{3},\{11\},\{22\}>\right\}$ and $\operatorname{let} A_{3}=<X_{3},\{22\},\{11\}>, B_{3}=<$ $X_{3},\{11\},\{22\}>$. Then $\operatorname{IFr}\left(A_{3}\right)=<X_{3},\{22\},\{11\}>$, $\operatorname{IFr}\left(B_{3}\right)=<X_{3},\{22\},\{11\}>$ and $\operatorname{IFr}\left(A_{3} \cap B_{3}\right)=\phi$ which implies $\operatorname{IFr}\left(A_{3}\right) \cap \operatorname{Icl}\left(B_{3}\right)=<X,\{22\},\{11\} \tilde{>}$ and $\operatorname{IFr}\left(B_{3}\right) \cap \operatorname{Icl}\left(A_{3}\right)=\phi$ implies $\operatorname{IFr}\left(A_{3} \cap\right.$ $\left.B_{3}\right) \subseteq\left(\operatorname{IFr}\left(A_{3}\right) \cap \operatorname{Icl}\left(B_{3}\right)\right) \cup\left(\operatorname{IFr}\left(\tilde{B_{3}}\right) \cap \operatorname{Icl}\left(A_{3}\right)\right) \quad$ but $\left(\operatorname{IFr}\left(A_{3}\right) \cap \operatorname{Icl}\left(B_{3}\right)\right) \cup\left(\operatorname{IFr}\left(B_{3}\right) \cap \operatorname{Icl}\left(A_{3}\right)\right) \nsubseteq \operatorname{IFr}\left(A_{3} \cap B_{3}\right)$.

Theorem:3.12. An intuitionistic continuous mapping be $h:\left(X_{1}, \mu\right) \rightarrow\left(Y_{1}, v\right)$ then

$\operatorname{IFr}\left(h^{-1}\left(B_{1}\right)\right) \subseteq h^{-1}\left(\operatorname{IFr}\left(B_{1}\right)\right)$ in any IS $B_{1}$ in $Y_{1}$.

Proof: Let $\mathrm{h}$ is intuitionistic continuous. $B_{1}$ be an IS in $Y_{1}$. Then

$\operatorname{IFr}\left(h^{-1}\left(B_{1}\right)\right)=\operatorname{Icl}\left(h^{-1}\left(B_{1}\right)\right) \cap \operatorname{Icl}\left(h^{-1}\left(B_{1}\right)\right)^{c}$

$\subseteq \operatorname{Icl}\left(h^{-1}\left(\operatorname{Icl}\left(B_{1}\right)\right)\right) \cap \operatorname{Icl}\left(h^{-1}\left(\operatorname{Icl}\left(B_{1}\right)^{c}\right)\right)$

$\subseteq h^{-1}\left(\operatorname{Icl}\left(B_{1}\right)\right) \cap h^{-1}\left(\operatorname{Icl}\left(B_{1}\right)^{c}\right)=h^{-1}\left(\operatorname{Icl}\left(B_{1}\right) \cap \operatorname{Icl}\left(B_{1}^{c}\right)\right)$ $\subseteq h^{-1}\left(\operatorname{IFr}\left(B_{1}\right)\right)$

Lemma : 3.13. Let $\mathrm{A}_{4}$ and $B_{4}$ are two intuitionistic sets, $A_{4} \subseteq B_{4}$ and $\mathrm{B}_{4}$ is $\operatorname{ICS}(X)$, then $\operatorname{IFr}\left(A_{4}\right) \subseteq B_{4}$.

Proof. Since $A_{4} \subseteq B_{4}$ implies $\operatorname{Icl}\left(A_{4}\right) \subseteq \operatorname{Icl}\left(B_{4}\right)$, which implies $\operatorname{IFr}\left(A_{4}\right)=\operatorname{Icl}\left(A_{4}\right) \cap \operatorname{Icl}\left(A_{4}^{c}\right) \subseteq$ $\operatorname{Icl}\left(B_{4}\right) \cap \operatorname{Icl}\left(B_{4}^{c}\right) \subseteq \operatorname{Icl}\left(B_{4}\right)=B_{4}$.

Theorem:3.14. Consider $h_{1}: X_{2} \rightarrow Y_{2}$ be an IO mapping, $B_{2}$ be an IS in $Y_{2}$. Then $h_{1}^{-1}\left(\operatorname{IFr}\left(B_{2}\right)\right) \subseteq$ $\operatorname{IFr}\left(h_{1}^{-1}\left(B_{2}\right)\right)$.

Proof:Suppose $h_{1}$ is an IO function, $B_{2}$ is an IS in $Y_{2}$. Let $A_{2}=\operatorname{Icl}\left(\operatorname{IFr}\left(h_{1}^{-1}\left(B_{2}\right)\right)\right)$. Then $A_{2}$ is IO, therefore $h_{1}\left(A_{2}\right)$ is $\mathrm{IO}$ in $Y_{2}$. This gives $\operatorname{Icl}\left(h_{1}\left(A_{2}\right)\right) \in \operatorname{ICS}\left(Y_{2}\right)$. This follows $\mathrm{B}_{2} \subseteq \operatorname{Icl}\left(h_{1}\left(A_{2}\right)\right)$. By Lemma 3.13, $h_{1}^{-1}\left(\operatorname{IFr}\left(B_{2}\right)\right) \subseteq h_{1}^{-1}\left(\operatorname{Icl}\left(h_{1}\left(A_{2}\right)\right)\right) \subseteq$ $\operatorname{Icl}\left(A_{2}\right)=\operatorname{Icl}\left(\operatorname{Icl}\left(\operatorname{IFr}\left(h_{1}^{-1}\left(B_{2}\right)\right)\right)\right)=\operatorname{IFr}\left(h_{1}^{-1}\left(B_{2}\right)\right)$. Consequently, $\operatorname{IFr}\left(h_{1}^{-1}\left(B_{2}\right)\right) \subseteq \operatorname{IFr}\left(h_{1}^{-1}\left(B_{2}\right)\right)$.

\section{Intuitionistic Semi Frontier}

Levine [6] generalized the notion of open sets as semiopen sets. The generalized work was helpful to develop a wider framework for the study of continuity and its different variants.

Definition:4.1. Consider $\left(X_{4}, \mu\right)$ be an $\operatorname{ITS}\left(X_{4}\right)$, $M \in I S\left(X_{4}\right)$. Also $q \in \operatorname{IFrP}\left(X_{4}\right)$ is defined as intuitionistic semifrontier point $(\operatorname{IsFrP})$ of $\mathrm{M}$ if $q \in \operatorname{Iscl}(M) \cap \operatorname{Iscl}\left(M^{c}\right)$. The union of all the IsFrPs of $\mathrm{M}$ is termed as an intuitionistic semifrontier of M. It can be noted as $(\operatorname{IsFr}(M))$. Also $\operatorname{IsFr}(M)=\operatorname{Iscl}(M) \cap \operatorname{Iscl}\left(M^{c}\right)$ holds.

Theorem: 4.2.For IS's $\mathrm{M}$ and $\mathrm{N}$ in an $\operatorname{ITS}\left(X_{4}\right)$,

(a) $\operatorname{Iscl}(\operatorname{Iscl}(M))=\operatorname{Iscl}(M)$

(b) $I \operatorname{sint}(I \operatorname{sint}(M))=I \operatorname{sint}(M)$

(c) $\operatorname{Isint}(M) \cup \operatorname{Isint}(N) \subseteq \operatorname{Isint}(M \cup N)$

(d) $\operatorname{Isint}(M \cap N)=\operatorname{Isint}(M) \cap I \operatorname{sint}(N)$

(e) $\operatorname{Iscl}(M \cup N)=\operatorname{Iscl}(M) \cup \operatorname{Iscl}(N)$

(f) $\operatorname{Iscl}(M \cap N) \subseteq \operatorname{Iscl}(M) \cap \operatorname{Iscl}(N)$.

Proof:(a) M is ISC [5] iff $M=\operatorname{Iscl}(M)$. Since $\operatorname{Iscl}(M)$ is ISC, $\operatorname{Iscl}(\operatorname{Iscl}(M))=\operatorname{Iscl}(M)$.

(b) Since $\operatorname{Isint}(M)$ is ISO and M is ISO iff $M=I \operatorname{sint}(M)$, therefore $I \operatorname{sint}(I \operatorname{sint}(M))=I \operatorname{sint}(M)$.

(c) $\operatorname{Isint}(M)$ and $I \operatorname{sint}(N)$ are both ISO sets and $\mathrm{M} \subseteq \mathrm{M} \cup \mathrm{N}, \quad \mathrm{N} \subseteq \mathrm{M} \cup \mathrm{N}$ implies $\operatorname{Isint}(M) \subseteq$ $\operatorname{Isint}(M \cup N)$ and $\operatorname{Isint}(N) \subseteq \operatorname{Isint}(M \cup N)$. This implies $\operatorname{Isint}(M) \cup \operatorname{Isint}(N) \subseteq$ Isint $(M \cup N)$.

(d) As $\mathrm{M} \cap N$ is an intuitionistic subset of $\mathrm{M}$ and $\mathrm{M} \cap N$ is an intuitionistic subset of $\mathrm{N}$ implies $\operatorname{Isint}(M \cap N) \subseteq$ $\operatorname{Isint}(M) \cap \operatorname{Isint}(N)$. Conversely $\operatorname{Isint}(M) \subseteq M$ and $\operatorname{Isint}(N) \subseteq \mathrm{N}$ implies $\operatorname{Isint}(M) \cap \operatorname{Isint}(N) \subseteq M \cap N$ and $\operatorname{Isint}(M) \cap \operatorname{Isint}(N)$ is an ISO set. But Isint $(M \cap N)$ is the biggest ISO set contained in $\mathrm{M} \cap \mathrm{N}$. Hence $\operatorname{Isint}(M) \cap \operatorname{Isint}(N) \subseteq M \cap N$. This gives the equality.

(e) Since $M$ is an intuitionistic subset of $M \cup N$ and $\mathrm{N}$ is an intuitionistic subset of $M \cup N, \operatorname{Iscl}(M) \subset$ 
$\operatorname{Iscl}(M \cup N)$ and $\operatorname{Iscl}(N) \subset \operatorname{Iscl}(M \cup N) \quad$ because $\mathrm{M} \subset \mathrm{N} \Rightarrow \operatorname{Iscl}(M) \subset \operatorname{Iscl}(N)$. Hence $\operatorname{Iscl}(M) \cup \operatorname{Iscl}(N) \subset$ $\operatorname{Iscl}(M \cup N) \longrightarrow(1)$.

Since $\operatorname{Iscl}(M), \operatorname{Iscl}(N)$ are ISC sets, $\operatorname{Iscl}(M) \cup \operatorname{Iscl}(N)$ is also intuitionistic closed. Also $M \subset \operatorname{Iscl}(M)$ and $N \subset \operatorname{Iscl}(N)$ implies that $M \cup N \subset \operatorname{Iscl}(M) \cup \operatorname{Iscl}(N)$. Since $\operatorname{Iscl}(M \cup N)$ is the smallest ISC set containing $M \cup N, \operatorname{Iscl}(M \cup N) \subset \operatorname{Iscl}(M) \cup \operatorname{Iscl}(N) \longrightarrow(2)$. From (1) and (2), $\operatorname{Iscl}(M \cup N)=\operatorname{Iscl}(M) \cup \operatorname{Iscl}(N)$.

(f) Hence $\mathrm{M} \cap N \subseteq M$ and $\mathrm{M} \cap N \subseteq N$ implies $\operatorname{Iscl}(M \cap N) \subseteq \operatorname{Iscl}(M)$ also $\operatorname{Iscl}(M \cap N) \subseteq \operatorname{Iscl}(N)$ implies $\operatorname{Iscl}(M \cap N) \subseteq \operatorname{Iscl}(M) \cap \operatorname{Iscl}(N)$.

Theorem:4.3. Let $M$ be an intuitionistic set in an $\operatorname{ITS}\left(X_{4}\right)$, the following statement holds.

(a) $\operatorname{IsFr}(M)=\operatorname{IsFr}\left(M^{c}\right)$.

(b) If $M_{1}$ is ISC, then $\operatorname{IsFr}\left(M_{1}\right) \subseteq M_{1}$.

(c) Suppose $M_{2}$ is ISO, then $\operatorname{IsFr}\left(M_{2}\right) \subseteq M_{2}^{c}$.

(d) Let $M \subseteq \mathrm{N}$ and $\mathrm{N} \in I S C(X)$ (resp. $\mathrm{N} \in I S O(X))$ then $\operatorname{IsFr}(M) \subseteq N$ (resp. $\left.\operatorname{IsFr}(M) \subseteq N^{c}\right)$ where $\operatorname{ISC}(X)$ (resp. $\mathrm{ISO}(X))$ denotes the ISC(ISO resp.) sets in X.

(e) $\left(\operatorname{IsFr}\left(M_{3}\right)\right)^{c}=\operatorname{Isint}\left(M_{3}\right) \cup \operatorname{Isint}\left(M_{3}^{c}\right)$.

(f) $\operatorname{IsFr}\left(M_{4}\right) \subseteq \operatorname{IFr}\left(M_{4}\right)$.

$(g) \operatorname{Iscl}(\operatorname{IsFr}(M)) \subseteq \operatorname{IFr}(M)$.

Proof:(a) Let $q \in \operatorname{IsFr}(M) \Leftrightarrow$ every intuitionistic neighbourhood (Inhd shortly)[2] of qintersects both $\mathrm{M}$ and $\mathrm{M}^{c} \Leftrightarrow$ every Inhd of $q$ intersects both $\left(M^{c}\right)^{c}$ and $M^{c}$, because $\left(M^{c}\right)^{c}=M \Leftrightarrow \tilde{q} \in \operatorname{IsFr}\left(M^{c}\right)$.

Proof of $(b),(c),(d)$ and $(e)$ are analogous of Theorem 3.8 .

(f) Since $\operatorname{Iscl}\left(M_{4}\right) \subseteq \operatorname{Icl}\left(M_{4}\right)$ and $\operatorname{Iscl}\left(M_{4}^{c}\right) \subseteq \operatorname{Icl}\left(M_{4}^{c}\right)$, then it gives $\operatorname{IsFr}\left(M_{4}\right)=\operatorname{Iscl}\left(M_{4}\right) \cap \operatorname{Iscl}\left(M_{4}^{c}\right) \subseteq$ $\operatorname{Icl}\left(M_{4}\right) \cap \operatorname{Icl}\left(M_{4}^{c}\right)=\operatorname{IFr}\left(M_{4}\right)$.

$(g) \operatorname{Iscl}(\operatorname{IsFr}(M))=\operatorname{Iscl}\left(\operatorname{Iscl}(M)\left(M^{c}\right)\right) \subseteq$

$\operatorname{Iscl}(\operatorname{Iscl}(M)) \cap \operatorname{Iscl}\left(\operatorname{Iscl}\left(M^{c}\right)\right)=\operatorname{Iscl}(\mathrm{M}) \cap \operatorname{Iscl}\left(M^{c}\right)=$ $\operatorname{IsFr}(M) \subseteq \operatorname{IFr}(M)$.

Example:4.4. Let $X_{4}=\left\{a_{4}, b_{4}, c_{4}\right\}$ with $\mu=\left\{\underset{\sim}{X}, \phi,<X_{4}, \phi,\left\{b_{4}, c_{4}\right\}>,<X_{4}, \phi,\left\{c_{4}\right\}>\right.$,

$\left.<X_{4},\left\{a_{4}, b_{4}\right\},\left\{c_{4}\right\}>,<X_{4},\left\{a_{4}, b_{4}\right\}, \phi>\right\}$,

(i) ISC sets are $\left\{X_{4}, \phi,<X_{4}, \phi, \phi>,<X_{4}, \phi,\left\{a_{4}\right\}>\right.$,

$<X_{4}, \phi,\left\{c_{4}\right\}>,<X_{4}, \phi,\left\{a_{4}, b_{4}\right\}>,<X_{4},\left\{b_{4}\right\},\left\{c_{4}\right\}>$,

$\left.<X_{4},\left\{c_{4}\right\}, \phi>,<X_{4},\left\{c_{4}\right\},\left\{b_{4}\right\}>,<X_{4},\left\{b_{4}, c_{4}\right\}, \phi>\right\} \quad$ and

$M_{1}=<X_{4}, \phi,\left\{c_{4}\right\}>, \operatorname{IsFr}\left(<X_{4}, \phi,\left\{c_{4}\right\}>\right)=$

$<X_{4}, \phi,\left\{b_{4}, c_{4}\right\}>$. This implies $\operatorname{IsFr}\left(M_{1}\right) \subseteq M_{1}$ but $M_{1} \nsubseteq \operatorname{IsFr}\left(M_{1}\right)$.

(ii) ISO sets are $\left\{X_{4}, \phi,<X_{4}, \phi, \phi>,<X_{4}, \phi,\left\{b_{4}\right\}>\right.$,

$<X_{4}, \phi,\left\{c_{4}\right\}>,<X_{4}, \phi,\left\{b_{4}, c_{4}\right\}>,<X_{4},\left\{c_{4}\right\},\left\{b_{4}\right\}>$,

$\left.<X_{4},\left\{c_{4}\right\}, \phi>,<X_{4},\left\{a_{4}, b_{4}\right\},\left\{c_{4}\right\}>,<X_{4},\left\{a_{4}, b_{4}\right\}, \phi>\right\}$,

IsFr $<X_{4}, \phi,\left\{b_{4}, c_{4}\right\}>=\left\langle X_{4}, \phi,\left\{b_{4}, c_{4}\right\}>\right.$ implies $\operatorname{IsFr}\left(<X_{4}, \phi,\left\{b_{4}, c_{4}\right\}>\right) \subseteq M_{2}^{c}$ but $M_{2}^{c} \nsubseteq \mathbb{I s F r}\left(M_{2}\right)$.

(iii) From Example 3.11., Iscl $(\operatorname{IsFr}(M))=\left\langle X_{3}, \phi,\{11\}>\right.$ implies $\operatorname{Iscl}(\operatorname{IsFr}(M)) \subseteq \operatorname{IFr}(M)$ but $\operatorname{IFr}(M) \nsubseteq$ $\operatorname{Iscl}(\operatorname{IsFr}(M))$. (iv) From Example 3.11, let $M=<X_{3},\{11\}, \phi>$, $\operatorname{IsFr}(M)=<X_{3}, \phi,\{11\}>, \operatorname{IFr}(M)=$

$<X_{3},\{22\},\{11\}>$ implies $\operatorname{IsFr}(M) \subseteq \operatorname{IFr}(M) \quad$ but $\operatorname{IFr}(M) \nsubseteq \operatorname{IsFr}(M)$.

Theorem: 4.5. Let $\mathrm{M}$ be an IS in an $\operatorname{ITS}\left(X_{4}\right)$. Then

(a) $\operatorname{IsFr}\left(M_{4}\right)=\operatorname{Iscl}\left(M_{4}\right)-\operatorname{Isint}\left(M_{4}\right)$.

(b) $\operatorname{IsFr}\left(\operatorname{Isint}\left(M_{4}\right)\right) \subseteq \operatorname{IsFr}\left(M_{4}\right)$.

(c) $\operatorname{IsFr}\left(\operatorname{Iscl}\left(M_{4}\right)\right) \subseteq \operatorname{IsFr}\left(M_{4}\right)$.

(d) $\operatorname{Isint}\left(M_{4}\right) \subseteq M_{4}-\operatorname{IsFr}\left(M_{4}\right)$.

Proof: (a) Let $\operatorname{IsFr}\left(M_{4}\right)=\operatorname{Iscl}\left(M_{4}\right) \cap \operatorname{Iscl}\left(M_{4}^{c}\right)=$ $\operatorname{Iscl}\left(M_{4}\right) \cap \operatorname{Iscl}\left(\underset{\sim}{X}-M_{4}\right)=\operatorname{Iscl}\left(M_{4}\right) \cap\left(\operatorname{Isint}\left(M_{4}\right)\right)^{c}=$ $\operatorname{Iscl}\left(M_{4}\right)-\operatorname{Isint}\left(M_{4}\right)$.

(b) $\operatorname{IsFr}\left(\operatorname{Isint}\left(M_{4}\right)\right)=\operatorname{Iscl}\left(\operatorname{Isint}\left(M_{4}\right)\right) \cap \operatorname{Iscl}\left(\operatorname{Isint}\left(M_{4}\right)^{c}\right)$ $\subseteq \operatorname{Iscl}\left(M_{4}\right) \cap \operatorname{Iscl}\left(M_{4}^{c}\right) \subseteq \operatorname{IsFr}\left(M_{4}\right)$.

(c) $\operatorname{IsFr}\left(\operatorname{Iscl}\left(M_{4}\right)\right)=\operatorname{Iscl}\left(\operatorname{Iscl}\left(M_{4}\right)\right) \cap \operatorname{Iscl}\left(\operatorname{Iscl}\left(M_{4}\right)^{c}\right) \subseteq$ $\operatorname{Iscl}\left(M_{4}\right) \cap \operatorname{Iscl}\left(M_{4}^{c}\right) \subseteq \operatorname{IsFr}\left(M_{4}\right)$, by Theorem 4.2 .

(d) Let $M_{4}-\operatorname{IsFr}\left(M_{4}\right)=M_{4}-\left(\operatorname{Iscl}\left(M_{4}\right)-\operatorname{Isint}\left(M_{4}\right)\right) \supseteq$ $\operatorname{Isint}\left(M_{4}\right)$.

Example:4.6.i) In Example 3.11, let $M_{4}=<X_{3}, \phi,\{1\}>$, $I \operatorname{sint}\left(M_{4}\right)=\phi, I s F r\left(\operatorname{Isint}\left(M_{4}\right)\right)=\phi$ and $\operatorname{IsFr}\left(M_{4}\right)=$

$<X_{3}, \phi,\{11\}>$. Thus $\operatorname{IsFr}\left(M_{4}\right) \nsubseteq \tilde{\operatorname{IsFr}}\left(\operatorname{Isint}\left(M_{4}\right)\right)$.

ii) Let $X_{5}=\left\{a_{5}, b_{5}, c_{5}\right\}, \mu=\left\{X_{5}, \phi,<X_{5}, \phi,\left\{c_{5}\right\}>\right.$,

$\left.<X_{5},\left\{c_{5}\right\}, \phi>,<X_{5},\left\{a_{5}, b_{5}\right\},\left\{c_{5}\right\}>,<X_{5}, \phi,\left\{b_{5}, c_{5}\right\}>\right\}$.

Let $M_{4}=<X_{5},\left\{c_{5}\right\},\left\{b_{5}\right\}>$, then $\operatorname{IsFr}\left(\operatorname{Iscl}\left(M_{4}\right)\right)=$

$<X_{5}, \phi,\left\{b_{5}, c_{5}\right\}>$, IsFr $\left(M_{4}\right)=<X_{5},\left\{c_{5}\right\},\left\{b_{5}\right\}>$. This implies $\operatorname{IsFr}\left(M_{4}\right) \nsubseteq \operatorname{IsFr}\left(\operatorname{Iscl}\left(M_{4}\right)\right)$.

iii) Let $X_{4}=\{12,22\}$ with intuitionistic topology $\mu=\left\{X_{4}, \phi,<X_{4},\{12\},\{22\}>,<X_{4}, \phi,\{22\}>\right\}$.

Let $\quad \tilde{M}_{4}=<X_{4}, \phi,\{12\}>$, then $\operatorname{Isint}\left(M_{4}\right)=\phi$. $M_{4}-\operatorname{IsFr}\left(M_{4}\right)=<X_{4}, \phi,\{12\}>$ This implies $M_{4}-\operatorname{IsFr}\left(M_{4}\right) \nsubseteq \operatorname{Isint}\left(M_{4}\right)$.

Theorem: 4.7. Let $M$ and N be IS's in an ITS $\left(X_{6}\right)$. Then $\operatorname{IsFr}(M \cup N) \subseteq \operatorname{IsFr}(M) \cup \operatorname{IsFr}(N)$.

Proof:Similar to Theorem 3.8. and converse of Theorem 4.7. need not be true.

Example:4.8.In an $\operatorname{ITS}\left(X_{6}\right)=\left\{a_{6}, b_{6}, c_{6}\right\}$ with IT $\mu=\left\{X_{6}, \phi,<X_{6},\left\{a_{6}\right\},\left\{b_{6}\right\}>,<X_{6},\left\{a_{6}, c_{6}\right\}, \phi>\right\}$ Let $\mathrm{M}=<\tilde{X}_{6},\left\{a_{6}\right\},\left\{b_{6}\right\}>, \quad \mathrm{N}=<X_{6},\left\{a_{6}, c_{6}\right\}, \phi>$. Then $\operatorname{IsFr}(M \cup N)=<X_{6}, \phi,\left\{a_{6}, c_{6}\right\}>$ and $\operatorname{IsFr}(M) \cup$ $\operatorname{Is} \operatorname{Fr}(N)=<X_{6},\left\{b_{6}\right\},\left\{a_{6}\right\}>$. This implies $\operatorname{IsFr}(M \cup N) \subseteq$ $\operatorname{IsFr}(M) \cup \operatorname{IsFr}(N) \quad$ but $\operatorname{IsFr}(M) \cup \operatorname{IsFr}(N) \nsubseteq$ $\operatorname{IsFr}(M \cup N)$.

\section{Conclusions}

In this paper, the development of intuitionistic frontier and its various properties in intuitionistic topological spaces are studied. Also notions of semifrontier in intuitionistic topology have been studied and several of their properties are proved. 


\section{References}

[1] K.T Atanassov, Intuitionistic fuzzy sets, Fuzzy Sets and Systems, 20, No.1 (1986), 87-66.

[2] D.Coker, A note on intuitionistic sets and intuitionistic points, Turkish J. Math., 20, No.3 (1996), 343-351.

[3] D.Coker, An introduction to intuitionistic fuzzy topological spaces, Fuzzy Sets and Systems, 88-1(1997), 81-89.

[4] D.Coker, An introduction to intuitionistic topological spaces, Busefal, 81(2000), 51-56.

[5] S.Girija and Gnanambal Ilango, Some More Results on Intuitionistic Semi Open Sets, International Journal of Engineering Research and Applications, 4(2014)11, 70-74.

[6] N. Levine, Semi open sets and Semi continuity in topological space, Amer. Math Monthly, 68(1961), 36-41.

[7] O.Njastad, On some classes of nearly open sets, Pacific J.Math, 15(1965), 961-970.

[8] A.A.Salama, Mohamed Abdelfattah and S.A.Alblowi, Some Intuitionistic Topological Notions of Intuitionistic
Region, Possible Application to GIS topological Rules, International Journal of Enhanced Research in Management Computer Applications, 3(2014)5, 4-9.

[9] S. Selvanayaki and Gnanambal Ilango, Strong and Weak forms of IGPR-continuity in intuitionistic topological spaces, International Journal of Pure and Applied Mathematics, 106(7)(2016), 45 - 55.

[10] S. Selvanayaki and Gnanambal Ilango, Generators in intuitionistic topological spaces, International Journal of Pure and Applied Mathematics, 116(2017)12, 957-966.

[11] Younis J.Yaseen and Asmaa G.Raouf , On generalization closed set and generalized continuity on intuitionistic topological spaces, J.of Al-anbar University of Pure Science, vol.3: no.1:(2009).

[12] Zadeh L.A, Fuzzy Sets, Information and Control, 8,(1965), 338-353. 